\title{
Atypical And Suspicious Categories in Fine Needle Aspiration Cytology of The Breast: Histopathological And Mammographical Correlation with Clinical Significance
}

\author{
Dr.G.Vandana ${ }^{1}$, Dr.S.Lokesh Rao Magar ${ }^{2}$, Dr. Sandhya Anil ${ }^{3}$ Dr. Sandhya \\ $\mathrm{Rani}^{4}$ \\ I assistant Professor In Pathology \\ 2 associate Professor In Pathology \\ ${ }^{3}$ professor In Pathology \\ ${ }^{4}$ professor \& Hod In Pathology
}

\begin{abstract}
Introduction: This study aims to correlate fine needle aspiration specimens diagnosed as C3 (atypical, probably benign) and C4 (suspicious, probably malignant) with histology and mammography, and to evaluate these two cytology categories in terms of diagnostic usefulness and patient management. Materials \& Methods: All fine-needle aspiration (FNA) specimens in categories $\mathrm{C} 3$ or C4 at the Mahatma Gandhi memorial Hospital, warangal between 2013-2015 were reviewed. Results were correlated with available histological and mammographical studies. Results: 289 FNA specimens were identified, comprising 84 category C3 and 205 category C4.Histology was available in 213 cases. 34 (64 percent) C3 cases showed benign histology on biopsy and 19 (36 percent) were malignant. 30 (19 percent) C4 cases were benign on biopsy, whereas 130 (81 percent) were malignant. Mammographical studies were available in 110 of the histologically proven cases. All 14 cases with benign mammograms had benign histology, and all 51 cases called "highly suggestive of malignancy" were malignant on histology (ten C3 and 41 C4). Of the 45 cases called "suspicious abnormality" on mammography, 27 turned out to be malignant on biopsy (two C3 and 25 C4). Conclusion: Our study supports maintaining cytology categories C3 and C4. About two-thirds of C3 cases were benign on biopsy whereas 81 percent of C4 cases were malignant ( $p$-value is less than 0.001). There was complete correlation between histological and mammographical studies except those with equivocal mammograms. Our study supports the combined use of clinical, mammographical and cytological findings for optimal patient management. This is especially important for patients with C3 aspiration results, in order to avoid unnecessary surgery for benign lesions.
\end{abstract}

Keywords: breast biopsy, breast cancer, cytology, fine-needle aspiration, mammography

\section{INTRODUCTION}

Fine-needle aspiration (FNA) of breast masses is widely accepted as a reliable diagnostic tool with both high sensitivity and specificity. Our previous study of over 2,000 cases showed a sensitivity of $84.4 \%$ and specificity of $99.5 \% \%^{1}$. Although, many centres now recommend needle core biopsy instead of FNA, we have advocated keeping FNA as a firstline diagnostic procedure, at least in areas under economic restriction ${ }^{1}$. Correlation of FNA results with the clinical and imaging studies is sometimes referred to as the "triple test $t^{2,3}$. Some studies have demonstrated $100 \%$ diagnostic accuracy using this approach ${ }^{2}$. The National Cancer Institute recommends five categories for the diagnosis of breast aspiration cytology ${ }^{4}$ in order to bring a degree of uniformity to the diagnostic reporting. These categories are: unsatisfactory $(\mathrm{C} 1)$, benign lesion $(\mathrm{C} 2)$, atypical, probably benign (C3), suspicious, probably malignant (C4) and malignant (C5). However, some authors believe that $\mathrm{C} 3$ and $\mathrm{C} 4$ should be categorised in the same group ${ }^{5}$. In order to investigate this hypothesis further, we reviewed our FNA cases in categories $\mathrm{C} 3$ and $\mathrm{C} 4$, and correlated the results with the available histological findings at subsequent surgery. Moreover, we correlated the cytology and histology results to the mammography findings to determine the accuracy of this test for breast lesions diagnosed in the $\mathrm{C} 3$ and $\mathrm{C} 4$ categories.

\section{Methods}

All FNAs performed at the Mahatma Gandhi memorial Hospital in warangal, Telangana between January 2013 and January 2015 were surveyed. Cases diagnosed as atypical, probably benign (C3) and suspicious, probably malignant $(\mathrm{C} 4)^{4}$ were selected for further study. Biopsies from subsequent operations on these cases were reviewed for correlation with the FNA results. Clinical data was obtained by chart review. The study was approved by the Research Ethics Board of the hospital.

Mammography studies were reviewed for the cases in the C3 and C4 categories that also had histological confirmation. All cases were reviewed by a breast radiologist. The mammographical studies were 
reported using the Breast Imaging Reporting and Data Systems (BI-RADS) that includes categories: BI-RADS 1 (negative lesion), BI-RADS 2 (benign lesion), BI-RADS 3 (probably benign), BIRADS 4 (suspicious abnormality), and BI-RADS 5 (highly suggestive of malignancy) ${ }^{6,7}$. Diagnostic value of cytological diagnoses and mammographical studies were assessed by comparing the percentage of benign or malignant histological diagnoses in categories $\mathrm{C} 3$ and $\mathrm{C} 4$ and by using the $\square$-square test of significance.

\section{Results}

From January 2013 to January 2015, 5,889 breast FNAs were performed at the Mahatma Gandhi memorial Hospital in warangal, Telangana. Out of 4,863 satisfactory specimens, 289 cases (5.9\%) were diagnosed as atypical, probably benign (C3) or suspicious, probably malignant (C4). All lesions in this study were palpable. The age range of the patients was 21 to 79 years. Subsequent operations were performed in 213 cases of those diagnosed in the C3 and C4 categories. Of those 213 cases, 110 cases had mammographical studies.

Table 1 :- Correlation Of Results Of Fna With Histology

\begin{tabular}{|l|l|l|l|l|}
\hline Cytological diagnosis & No. of cases & $\begin{array}{l}\text { No. of cases with } \\
\text { histology }\end{array}$ & $\begin{array}{l}\text { No. of cases with benign } \\
\text { histology }\end{array}$ & $\begin{array}{l}\text { No. of cases with malign } \\
\text { histology }\end{array}$ \\
\hline C3 & 84 & $53(51 \%)$ & $34(64 \%)$ & $19(36 \%)$ \\
C4 & 205 & $30(19 \%)$ & $130(81 \%)$ \\
\hline
\end{tabular}

OF the 289 cases selected for review, 84 (29\%) cases were category C3 and 205 (71\%) cases were category C4. Histology was available in 53 cases ofcategory C3 and 160 cases of category C4(Table I). About $34(64 \%)$ of cases in category C3 had final histological diagnoses of benign lesions, whereas only 19 (36\%) of cases in category $\mathrm{C} 4$ group did. The benign histological diagnoses for cases in category $\mathrm{C} 3$ included: fibroadenoma (21), papilloma (3), fibrocystic changes (4), gynaecomastia (2), lactational change (2), and epithelial hyperplasia (2). For the C4 category, the benign histological diagnoses included: fibroadenoma (13), fibrocystic change (9), papilloma (6) and granulomatous inflammation (2).

Malignancy was found in 19(36\%) of cases in category C3 but in 130( over 80\%) of cases in category C4. All malignant diagnoses were invasive ductal carcinoma except three cases of invasive lobular carcinoma in category $\mathrm{C} 4$.

There was a significant difference between the number of benign and malignant diagnoses for cytologic categories C3 and C4 ( $<0.001)$. Individual cases with histological correlation are shown in Figures

Figure -1 \& 2 shows monomorphic pattern with loosely cohesive clusters from categories which on histopathology is benign, while a malignant case from category $\mathrm{C} 4$ is shown in Fig. 3.

Fig 1

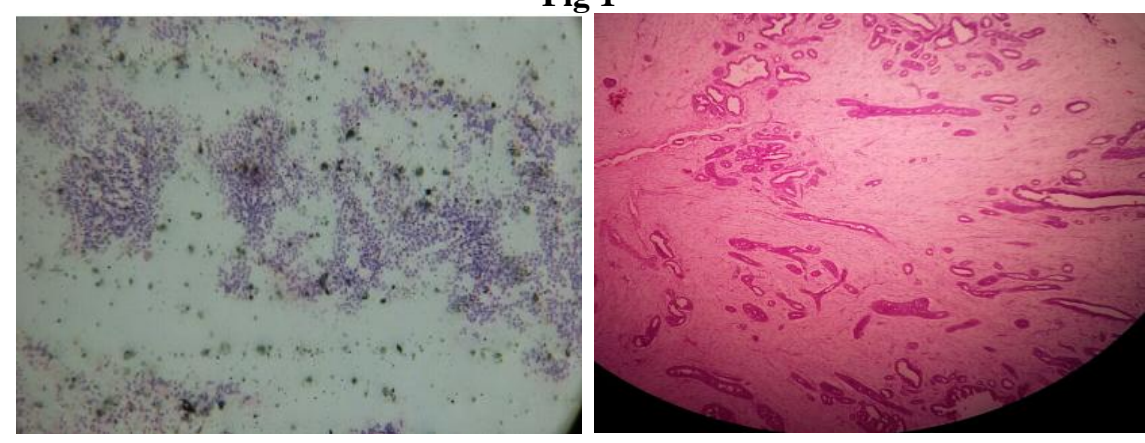

Fig 2

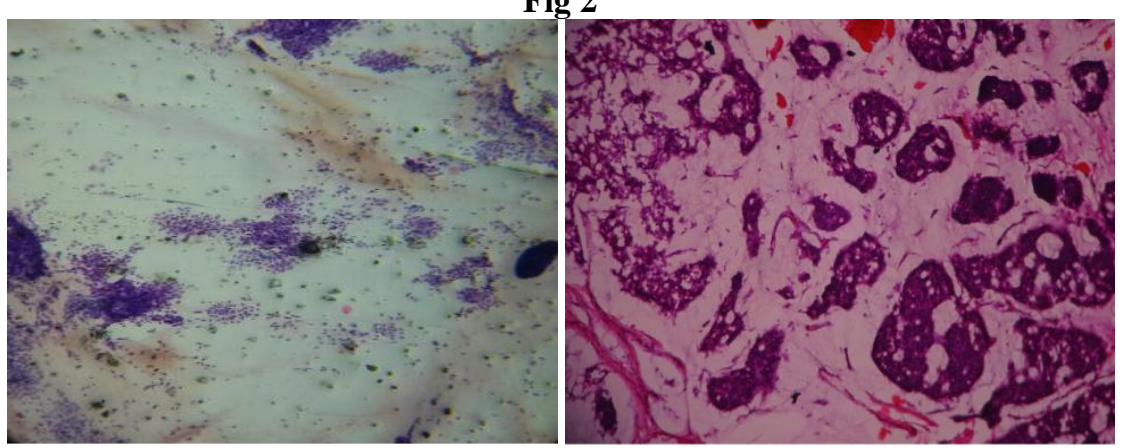


Fig 3
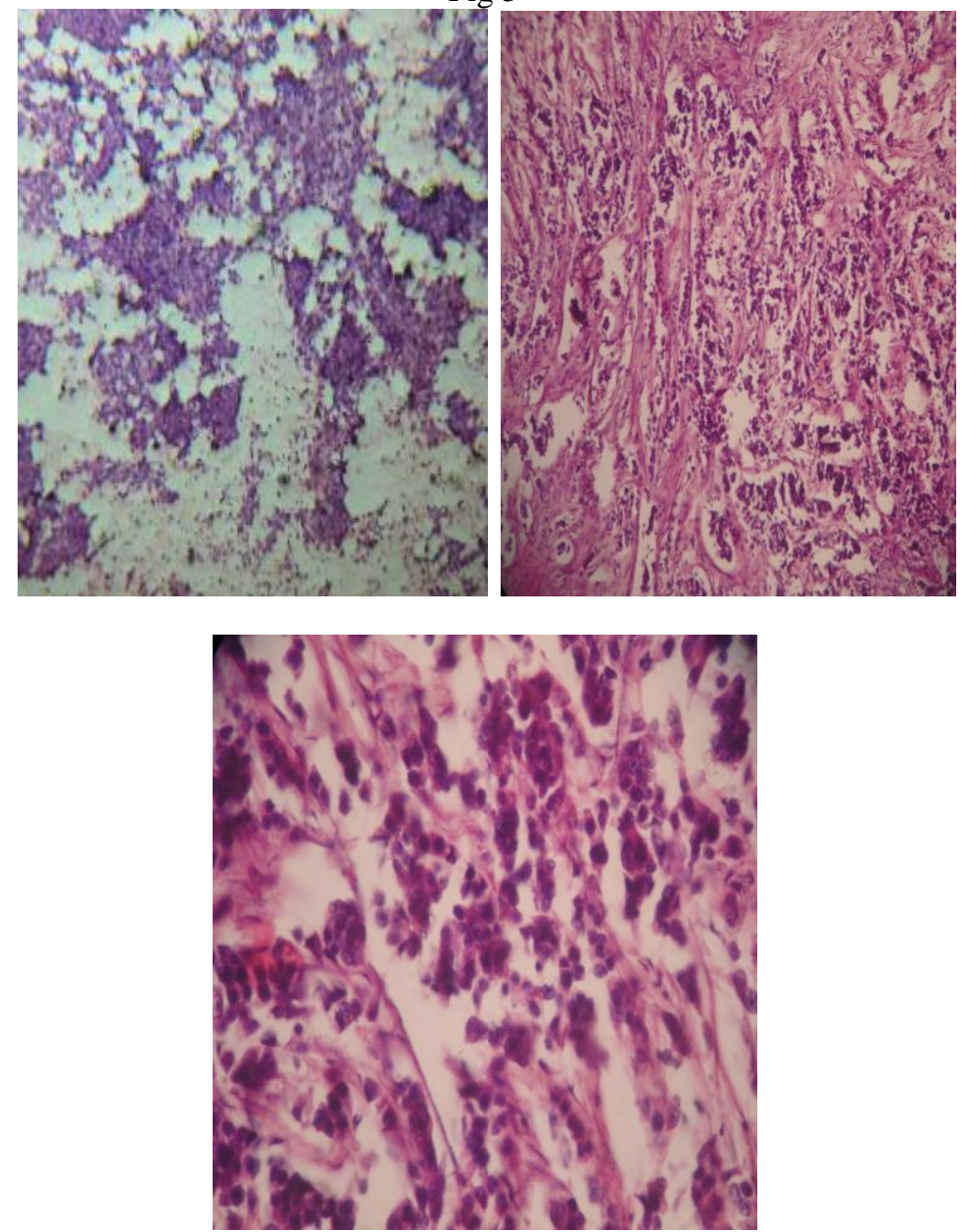

The correlation between the mammography, cytology and histology results is presented in Table II. All fourteen cases in the BI-RADS 1, 2 and 3 categories(negative, benign, probably benign) had benign histology on biopsy and were in cytology category C3. Of the 45 cases in category BI-RADS 4 (suspicious abnormality), 27 $(60.9 \%)$ had malignancy on biopsy, with two of these cases in cytology category C3 and the other 25 in cytology category C4. All 51 cases in category BI-RADS 5 (highly suggestive of malignancy) had malignant diagnoses on biopsy, with 10 cases in cytology category C3 and 41cases in cytology category C4.

Table II. Correlation of results of mammography with histology.

\begin{tabular}{|l|c|c|c|c|c|}
\hline Mammography results & No. of cases & \multicolumn{2}{c|}{ C3 cases with histology } & \multicolumn{2}{c|}{ C4 cases with histology } \\
\cline { 2 - 6 } & & benign & malignant & Benign & malignant \\
\hline BI-RADS 1 & 1 & 1 & 0 & 0 & 0 \\
BI-RADS 2 & 6 & 3 & 0 & 3 & 0 \\
BI-RADS 3 & 7 & 7 & 0 & 0 & 0 \\
BI-RADS 4 & 45 & 4 & 2 & 14 & 25 \\
& 51 & 0 & 10 & 0 & 41 \\
& & & & & \\
\hline
\end{tabular}

\section{Discussion}

From our study based on over 289 cases of breast FNA, the equivocal diagnostic categories C3 and C4 comprised approximately $5.9 \%$ of the total. This proportion is in the range (4-17.7\%) reported by others, ${ }^{5,8-13}$, indicating these categories are not being under used or over used in our centre. The diagnosis of category $\mathrm{C} 4$ was almost 2.5 times as common as $\mathrm{C} 3$. In this study, the three most common lesions contributing to the diagnosis of categories C3 and C4 were fibroadenoma, fibrocystic change and papilloma, which is similar to 
what has been reported by others ${ }^{5,10,14,15}$. The other less common lesions included: lactating adenoma, nonspecific inflammatory process, gynaecomastia, and granulomatous inflammation.

The most common malignant lesion diagnosed as category C3 or C4 was invasive ductal carcinoma, particularly with low nuclear grade. Occasionally, lesions were placed in the equivocal categories C3 and C4 because of technical factors such as air drying effect, bloody background and small number of malignant cells. This problem is generally avoided in our FNA clinic, in which specimens are evaluated immediately at the time of the aspiration, and any unsatisfactory specimens are repeated during the same procedure.

Of the cases in category $\mathrm{C} 3$ that proceeded to surgery, over $35 \%$ had a malignant diagnosis on biopsy. This result is also in line with reported literature values that range between $8.6 \%$ to $52 \%$, with most reports having over $30 \%^{5,8,9,12,16}$. The decision to proceed to biopsy in our centre relies, whenever possible, on the "triple test" 2,3 correlating the FNA results with the clinical and radiological studies. With this approach, the cases in category C3 that had clinical and imaging studies suspicious for malignancy were recommended for biopsy, thereby accounting for the seemingly high proportion of malignant cases in this category. Our study supports the recommendations of others ${ }^{17}$ that the use of the "triple test" is important for proper management of patients with FNA results in category $\mathrm{C} 3$, and whether such patients need to proceed to surgery, or can simply be followed. For cases in the C4 category, our study showed that approximately $80 \%$ of patient turned out to have malignant lesions, which is similar to the range of $81 \%$ to $97 \%$ reported by others $5,8,9,12,16$. Thus, as have been recommended by some authors ${ }^{5,18}$, we believe that patients in cytology category $\mathrm{C} 4$ should have the diagnosis confirmed by histological examination. Despite this recommendation, in our study, only $65 \%$ of patients in category $\mathrm{C} 4$ had surgery performed.

The remainder may have had the operation done in other hospitals, particularly private hospitals. Considering mammography and cytology together, it would appear that both techniques are equally accurate in predicting benign versus malignant diseases for lesions that are in the mammographical categories of BI-RADS 1-3. For lesions in category BI-RADS 4, cytology was a better predictor of which lesions were benign or malignant. Only 27 out of 45 cases in this category were actually malignant, but 25 of those 27 cases were in cytology category $\mathrm{C} 4$. For lesions in the radiological category BI-RADS 5,mammography was the better predictor since all those cases were malignant on biopsy, yet almost $20 \%$ of those cases were in cytology category $\mathrm{C} 3$.

\section{Conclusion}

It is important for both pathologists and clinicians to understand not only the benefits but also the limitations of cytological diagnosis from FNA specimens. Immediate evaluation of specimen adequacy is useful to eliminate equivocal diagnoses caused by technical factors. We feel it is still useful to maintain the equivocal diagnostic categories $\mathrm{C} 3$ and $\mathrm{C} 4$, since in our study, approximately two-thirds of patients who were diagnosed as category $\mathrm{C} 3$ had benign lesions, whereas about $80 \%$ of those in category $\mathrm{C} 4$ had malignant diagnoses. This difference was statistically significant $(\mathrm{p}<0.001)$. Combining the mammography and cytology data together with the clinical findings (the "triple test") allow for proper management for each patient, determining which should proceed to surgery and which can be closely followed, hence avoiding an unnecessary operation.

\section{References}

[1]. Chaiwun B, Settakorn J, Ya-In C, et al. Effectiveness of fine-needle aspiration cytology of breast: analysis of 2,375 cases from northern Thailand. Diagn Cytopathol 2002; 26:201-5.

[2]. Hermansen C, Skovgaard Poulsen H, Jensen J, et al. Palpable breast tumours: "triple diagnosis" and operative strategy. Results of a prospective study. Acta Chir Scand 1984; 150:625-8.

[3]. Layfield LJ, Glasgow BJ, Cramer H. Fine-needle aspiration in the management of breast masses. Pathol Annu 1989; 24 (pt 2):2362.

[4]. The uniform approach to breast fine-needle aspiration biopsy. National Cancer Institute Fine-Needle Aspiration of Breast Workshop Subcommittees. Diagn Cytopathol 1997; 16:295-311.

[5]. Kanhoush R, Jorda M, Gomez-Fernandez C, et al. 'Atypical' and 'suspicious' diagnoses in breast aspiration cytology. Cancer 2004; 102:164-7.

[6]. D'Orsi C. The American College of Radiology mammography lexicon: an initial attempt to standardize terminology. Am J Roentgenol 1996; 166:779-80.

[7]. Illustrated breast imaging reporting and data system (ACR BIRADS). 4th ed. Reston, VA: American College of Radiology; 2003.

[8]. Nicosia SV, Williams JA, Horowitz SA, et al. Fine needle aspiration biopsy of palpable breast lesions. Review and statistical analysis of 1875 cases. Surg Oncol 1993; 2:145-60.

[9]. Mulford D, Dawson AE. Atypia in fine needle aspiration cytology of nonpalpable and palpable mammographically detected breast lesions. Acta Cytol 1994; 38:9-17.

[10]. Willis SL, Ramzy I. Analysis of false results in a series of 835 fine needle aspirates of breast lesions. Acta Cytol 1995; 39:858-64.

[11]. Kim A, Lee J, Choi JS, et al. Fine needle aspiration cytology of the breast. Experience at an outpatient breast clinic. Acta Cytol 2000; 44:361-7.

[12]. Deb RA, Matthews P, Elston CW, et al. An audit of "equivocal" (C3) and "suspicious" (C4) categories in fine needle aspiration cytology of the breast. Cytopathology 2001; 12:219-26.

[13]. Lim J, Al-Masri H, Salhadar A, et al. The significance of the diagnosis of atypia in breast fine-needle aspiration. Diagn Cytopathol $2004 ; 31: 285-8$ 
[14]. Ariga R, Bloom K, Reddy VB, et al. Fine-needle aspiration of clinically suspicious palpable breast masses with histopathologic correlation. Am J Surg 2002; 184:410-3.

[15]. McKee GT. Reporting of breast cytology specimen. In: McKee GT, ed. Cytopathology of the Breast. Hong Kong: Oxford University Press, 2002: 58-82.

[16]. Wang HH, Ducatman BS. Fine needle aspiration of the breast. A probabilistic approach to diagnosis of carcinoma. Acta Cytol 1998; 42:285-9.

[17]. Logrono R, Kurtycz DF, Inhorn SL. Criteria for reporting fine needle aspiration on palpable and nonpalpable masses of the breast. Acta Cytol 1997; 41:623-7.

[18]. Muslumanoglu M, Dolay K, Ozmen V, et al. Comparison of fine needle aspiration cytology and excisional biopsy in palpable breast cancers. Radiol Med (Torino) 1995; 89:225-8 